\title{
Perturbative analysis of the gradient flow in non-abelian gauge theories
}

\author{
Martin Lüscher ${ }^{a}$ and Peter Weisz ${ }^{b}$ \\ ${ }^{a}$ CERN, Physics Department, \\ 1211 Geneva 23, Switzerland \\ ${ }^{b}$ Max-Planck-Institut für Physik, \\ Föhringer Ring 6, 80805 Munich, Germany \\ E-mail: luscher@mail.cern.ch, pew@mpp.mpg.de
}

ABSTRACT: The gradient flow in non-abelian gauge theories on $\mathbb{R}^{4}$ is defined by a local diffusion equation that evolves the gauge field as a function of the flow time in a gaugecovariant manner. Similarly to the case of the Langevin equation, the correlation functions of the time-dependent field can be expanded in perturbation theory, the Feynman rules being those of a renormalizable field theory on $\mathbb{R}^{4} \times[0, \infty)$. For any matter multiplet and to all loop orders, we show that the correlation functions are finite, i.e. do not require additional renormalization, once the theory in four dimensions is renormalized in the usual way. The flow thus maps the gauge field to a one-parameter family of smooth renormalized fields.

Keywords: Lattice QCD, Lattice Gauge Field Theories, Renormalization Regularization and Renormalons, Field Theories in Higher Dimensions

ARXIV EPRINT: 1101.0963 


\section{Contents}

1 Introduction 1

2 Iterative solution of the flow equation $\quad 2$

2.1 Definition of the gradient flow 2

2.2 Expansion in powers of the fundamental gauge field 2

2.3 Flow-line diagrams 3

3 Perturbation theory $\quad 4$

3.1 Gauge fixing 4

3.2 Gauge-field propagator 5

3.3 Feynman rules in $D+1$ dimensions $\quad 6$

4 Field theory $D+1$ dimensions $\quad 7$

$\begin{array}{lll}4.1 \text { Action } & 7\end{array}$

4.2 Propagators 8

4.3 Vertices 8

4.4 Flow lines and flow-line loops 9

5 Sample calculation at one-loop order $\quad 9$

$\begin{array}{lr}5.1 \text { Computation of self-energy diagrams } & 9\end{array}$

$\begin{array}{ll}5.2 \text { Renormalization } & 11\end{array}$

5.3 Do the bulk fields require renormalization? 11

6 BRS symmetry 12

$\begin{array}{lll}\text { 6.1 BRS transformation of the boundary fields } & 12\end{array}$

$\begin{array}{ll}6.2 \text { Bulk ghost fields } & 13\end{array}$

$\begin{array}{lll}\text { 6.3 BRS variation of the fields in the bulk } & 14\end{array}$

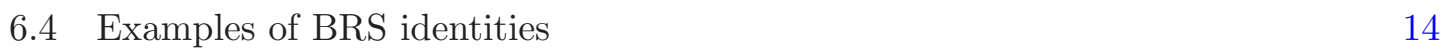

$\begin{array}{lll}7 & \text { Finiteness of the renormalized perturbation expansion } & \mathbf{1 5}\end{array}$

$\begin{array}{lll}7.1 & \text { Renormalized perturbation theory } & 15\end{array}$

$\begin{array}{ll}7.2 & \text { Absence of bulk counterterms } \\ \end{array}$

$\begin{array}{lll}7.3 & \text { Boundary counterterms } & 16\end{array}$

$\begin{array}{ll}7.4 \text { Consequences of the BRS symmetry } & 17\end{array}$

8 Miscellaneous remarks $\quad 18$

$\begin{array}{ll}\text { 8.1 Behaviour of the gauge field near flow time zero } & 18\end{array}$

$\begin{array}{ll}8.2 \text { Gauge-invariant composite fields } & 18\end{array}$

$\begin{array}{lll}8.3 & \text { Theories with matter fields } & 19\end{array}$

$\begin{array}{ll}8.4 \text { Lattice regularization } & 19\end{array}$ 


\section{Introduction}

The physics described by non-abelian gauge theories can be studied in many ways. Depending on the context and the questions to be answered, the desired information is extracted from correlation functions of local fields, expectation values of Wilson loops or the Schrödinger functional, for example.

As explained in refs. [1, 2], the gradient flow provides further opportunities to probe these theories and allows some of their otherwise elusive properties to be understood (in lattice gauge theory, the gradient flow is referred to as the Wilson flow, while in the mathematical literature it is commonly known as the Yang-Mills gradient flow). Evidently, physically meaningful probes must be safe of ultra-violet divergences or must be such that these can be canceled by a well-defined renormalization procedure.

In the case of the gradient flow, there is some evidence that the gauge field generated by the flow does not require renormalization $[1,2]$, but a formal proof of the absence of ultraviolet divergences at positive flow time has not been given so far. The aim of the present paper is to fill this gap through an all-order analysis of the flow in perturbation theory. With respect to the closely related case of the renormalization of the Langevin equation discussed by Zinn-Justin and Zwanziger [3, 4], there are two important differences, one being the absence of the noise term in the flow equation and the other the fact that the initial distribution of the gauge field (which is given by the functional integral of the theory considered) is not ignored.

The perturbative analysis presented in this paper applies to renormalizable gauge theories with any compact simple gauge group and any matter multiplet. However, in order to simplify the discussion as much as possible, only the pure $\mathrm{SU}(N)$ gauge theory with dimensional regularization will be considered, the generalization to other cases being straightforward (see section 8).

In the following three sections, the Feynman rules for the correlation functions of the gauge field generated by the gradient flow are derived and are shown to be those of a local field theory with an extra dimension (the flow time). The finiteness of the correlation functions at positive flow time can then be established using power-counting and the BRS symmetry (sections 6, 7), but for illustration the divergent parts of a set of one-loop diagrams are first worked out in section 5 . 


\section{Iterative solution of the flow equation}

In this section, we introduce the gradient flow and derive the Feynman rules for the associated flow-line diagrams. The pure $\mathrm{SU}(N)$ gauge theory in $D=4-2 \epsilon$ euclidean dimensions is considered and the fundamental gauge field $A_{\mu}(x)$ is normalized so that its action at bare coupling $g_{0}$ is given by

$$
\begin{aligned}
S & =-\frac{1}{2 g_{0}^{2}} \int \mathrm{d}^{D} x \operatorname{tr}\left\{F_{\mu \nu}(x) F_{\mu \nu}(x)\right\}, \\
F_{\mu \nu} & =\partial_{\mu} A_{\nu}-\partial_{\nu} A_{\mu}+\left[A_{\mu}, A_{\nu}\right]
\end{aligned}
$$

(see appendix A for unexplained notation).

\subsection{Definition of the gradient flow}

The gradient flow evolves the gauge field as a function of a parameter $t \geq 0$ that is referred to as the flow time. Starting from the fundamental gauge field,

$$
\left.B_{\mu}\right|_{t=0}=A_{\mu}
$$

the time-dependent field $B_{\mu}(t, x)$ is determined by the differential equation

$$
\begin{aligned}
\partial_{t} B_{\mu} & =D_{\nu} G_{\nu \mu}+\alpha_{0} D_{\mu} \partial_{\nu} B_{\nu} \\
G_{\mu \nu} & =\partial_{\mu} B_{\nu}-\partial_{\nu} B_{\mu}+\left[B_{\mu}, B_{\nu}\right], \quad D_{\mu}=\partial_{\mu}+\left[B_{\mu}, \cdot\right] .
\end{aligned}
$$

The name "gradient flow" derives from the fact that first term on the right of eq. (2.4) is proportional to the gradient of the gauge action along the flow. Note that neither the initial condition (2.3) nor the flow equation (2.4) involve the gauge coupling.

The second term on the right of eq. (2.4) is included in order to damp the evolution of the gauge degrees of freedom of the field. As in the case of the Langevin equation [4], some technicalities in the perturbative analysis of the flow can be avoided in this way without affecting the evolution of the gauge-invariant observables. The latter are in fact independent of the parameter $\alpha_{0}$, since the solutions of eq. (2.4) obtained at different values of $\alpha_{0}$ are related by a (time-dependent) gauge transformation [1].

\subsection{Expansion in powers of the fundamental gauge field}

Equation (2.4) may be split into a linear and remainder part according to

$$
\begin{aligned}
\partial_{t} B_{\mu} & =\partial_{\nu} \partial_{\nu} B_{\mu}+\left(\alpha_{0}-1\right) \partial_{\mu} \partial_{\nu} B_{\nu}+R_{\mu} \\
R_{\mu} & =2\left[B_{\nu}, \partial_{\nu} B_{\mu}\right]-\left[B_{\nu}, \partial_{\mu} B_{\nu}\right]+\left(\alpha_{0}-1\right)\left[B_{\mu}, \partial_{\nu} B_{\nu}\right]+\left[B_{\nu},\left[B_{\nu}, B_{\mu}\right]\right] .
\end{aligned}
$$

The linearized equation can be solved using the heat kernel

$$
K_{t}(z)_{\mu \nu}=\int_{p} \frac{\mathrm{e}^{i p z}}{p^{2}}\left\{\left(\delta_{\mu \nu} p^{2}-p_{\mu} p_{\nu}\right) \mathrm{e}^{-t p^{2}}+p_{\mu} p_{\nu} \mathrm{e}^{-\alpha_{0} t p^{2}}\right\}
$$


where

$$
\int_{p}=\int \frac{\mathrm{d}^{D} p}{(2 \pi)^{D}}
$$

Taking the boundary condition (2.3) into account, the flow equation may then be cast in the integral form

$$
B_{\mu}(t, x)=\int \mathrm{d}^{D} y\left\{K_{t}(x-y)_{\mu \nu} A_{\nu}(y)+\int_{0}^{t} \mathrm{~d} s K_{t-s}(x-y)_{\mu \nu} R_{\nu}(s, y)\right\} .
$$

From this representation the retarded character of the equation is evident and it is also quite clear that the sensitivity to the initial value of the field dies away as $t$ increases, although only slowly so at small momenta ( $\alpha_{0}$ is assumed to be positive).

When passing to momentum space,

$$
B_{\mu}(t, x)=\int_{p} \mathrm{e}^{i p x} \tilde{B}_{\mu}(t, p),
$$

the integral equation (2.10) becomes

$$
\tilde{B}_{\mu}(t, p)=\tilde{K}_{t}(p)_{\mu \nu} \tilde{A}_{\nu}(p)+\int_{0}^{t} \mathrm{~d} s \tilde{K}_{t-s}(p)_{\mu \nu} \tilde{R}_{\nu}(s, p) .
$$

It is helpful at this point to introduce the vertices $X^{(2,0)}$ and $X^{(3,0)}$ through

$$
\begin{aligned}
\tilde{R}_{\mu}^{a}(t, p)= & \sum_{n=2}^{3} \frac{1}{n !} \int_{q_{1}} \ldots \int_{q_{n}}(2 \pi)^{D} \delta\left(p+q_{1}+\ldots+q_{n}\right) \\
& \times X^{(n, 0)}\left(p, q_{1}, \ldots, q_{n}\right)_{\mu \nu_{1} \ldots \nu_{n}}^{a b_{1} \ldots b_{n}} \tilde{B}_{\nu_{1}}^{b_{1}}\left(t,-q_{1}\right) \ldots \tilde{B}_{\nu_{n}}^{b_{n}}\left(t,-q_{n}\right)
\end{aligned}
$$

and the requirement that they are totally symmetric in the momentum-index combinations $\left(q_{1}, \nu_{1}, a_{1}\right), \ldots,\left(q_{n}, \nu_{n}, a_{n}\right)$. The solution of the integral equation in powers of the fundamental gauge field,

$$
\begin{aligned}
\tilde{B}_{\mu}^{a}(t, p)= & \tilde{K}_{t}(p)_{\mu \nu} \tilde{A}_{\nu}^{a}(p)+\frac{1}{2} \int_{0}^{t} \mathrm{~d} s \tilde{K}_{t-s}(p)_{\mu \nu} \int_{q, r}(2 \pi)^{D} \delta(p-q-r) \\
& \times X^{(2,0)}(p,-q,-r)_{\nu \rho \sigma}^{a b c} \tilde{K}_{s}(q)_{\rho \delta} \tilde{K}_{s}(r)_{\sigma \tau} \tilde{A}_{\delta}^{b}(q) \tilde{A}_{\tau}^{c}(r)+\ldots
\end{aligned}
$$

is then obtained through iteration, i.e. by recursively inserting the equation on the right of itself.

The vertices $X^{(2,0)}$ and $X^{(3,0)}$ are given explicitly in appendix B. Note that the momentum-index combination $(p, \mu, a)$ plays a special rôle in eq. (2.13). In particular, the vertices are symmetric only in their other arguments.

\subsection{Flow-line diagrams}

The terms contributing to the expansion (2.14) in powers of the fundamental gauge field can be graphically represented by Feynman diagrams (see figure 1). There are two kinds 


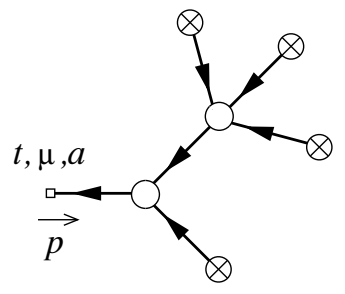

Figure 1. The diagrams contributing to $\tilde{B}_{\mu}^{a}(t, p)$ are directed tree graphs with a single external line (a little square is drawn at the end of this line). Flow lines always start from a one-point vertex (circle with a cross) or a flow vertex (circle) and end at another flow vertex if the line is not the external one. Each flow vertex has one outgoing flow line that corresponds to the momentum-index combination $(p, \mu, a)$ in eq. (2.13).

of vertices in these diagrams, the flow vertices $X^{(2,0)}$ and $X^{(3,0)}$ introduced in the previous subsection and the one-point vertex

$$
\underset{p}{\stackrel{\mu}{\rightarrow} \leftarrow \otimes}=\tilde{A}_{\mu}^{a}(p)
$$

Each flow vertex is inserted at some flow time that is integrated from zero to infinity, while the one-point vertices reside at time zero. The vertices are connected through the flow lines

$$
\stackrel{t, \mu, a \underset{p}{\longleftarrow} s, v, b}{\longleftarrow}=\delta^{a b} \theta(t-s) \tilde{K}_{t-s}(p)_{\mu \nu},
$$

where $t$ and $s$ are the flow times at the endpoints of the line. In view of the retarded nature of the propagator (2.16), the flow time increases from zero at the one-point vertices to time $t$ at the outer end of the external line as one follows the arrows in the diagram. In particular, the times associated with the vertices are effectively integrated only up to $t$ (rather than infinity).

As far the momenta, the index contractions and the symmetry factors are concerned, the Feynman rules are the usual ones. It is then not difficult to show that the sum of all diagrams solves the flow equation (2.12) order by order in the fundamental gauge field.

\section{Perturbation theory}

The $n$-point correlation functions of the field $B_{\mu}^{a}(t, x)$ can be computed in perturbation theory by expanding the field in powers of the fundamental field, as explained section 2, and by calculating the correlation functions of the latter in the $\mathrm{SU}(N)$ gauge theory as usual. We now show that the correlation functions can be directly obtained from a set of Feynman rules in $D+1$ dimensions.

\subsection{Gauge fixing}

The flow equation (2.4) is invariant under the infinitesimal transformation

$$
\delta B_{\mu}=D_{\mu} \omega
$$


provided the time-dependence of $\omega(t, x) \in \mathfrak{s u}(N)$ is such that

$$
\partial_{t} \omega=\alpha_{0} D_{\mu} \partial_{\mu} \omega
$$

Since the initial value of $\omega$ is unconstrained, these transformations generate the full gauge group at flow time $t=0$ and thus extend the gauge symmetry of the $\mathrm{SU}(N)$ gauge theory to all flow times.

The symmetry can be fixed as usual by including the gauge-fixing term

$$
S_{\text {gf }}=-\frac{\lambda_{0}}{g_{0}^{2}} \int \mathrm{d}^{D} x \operatorname{tr}\left\{\partial_{\mu} A_{\mu}(x) \partial_{\nu} A_{\nu}(x)\right\}
$$

and the associated ghost action

$$
S_{c \bar{c}}=-\frac{2}{g_{0}^{2}} \int \mathrm{d}^{D} x \operatorname{tr}\left\{\partial_{\mu} \bar{c}(x) D_{\mu} c(x)\right\}
$$

in the total action of the theory, where $c$ and $\bar{c}$ are the Faddeev-Popov ghost fields. As far as the $n$-point correlation functions of the fundamental gauge field and the ghosts are concerned, the Feynman rules are then the standard ones. Evidently, since the transformation (3.1) is an infinitesimal gauge variation, the expectation values of gauge-invariant expressions in the field generated by the flow are independent of the gauge parameter $\lambda_{0}$.

\subsection{Gauge-field propagator}

The way in which the flow-line diagrams combine with the Feynman diagrams of the underlying theory is best explained by considering the two-point function of the timedependent gauge field. To leading order in the gauge coupling, the flow-line diagram

$$
\stackrel{t, \mu, a}{\vec{p}} \longrightarrow \underset{\stackrel{\leftarrow}{\leftarrow}}{\stackrel{s, v, b}{\leftarrow}}
$$

is the only one that contributes to the correlation function. The contraction of the gauge fields at the one-point vertices then shows that

$$
\begin{aligned}
\left\langle\tilde{B}_{\mu}^{a}(t, p) \tilde{B}_{\nu}^{b}(s, q)\right\rangle & =(2 \pi)^{D} \delta(p+q) \delta^{a b} g_{0}^{2} \tilde{D}_{t+s}(p)_{\mu \nu}+\mathrm{O}\left(g_{0}^{4}\right) \\
\tilde{D}_{t}(p)_{\mu \nu} & =\frac{1}{\left(p^{2}\right)^{2}}\left\{\left(\delta_{\mu \nu} p^{2}-p_{\mu} p_{\nu}\right) \mathrm{e}^{-t p^{2}}+\lambda_{0}^{-1} p_{\mu} p_{\nu} \mathrm{e}^{-\alpha_{0} t p^{2}}\right\} .
\end{aligned}
$$

This formula includes the mixed propagator

$$
\left\langle\tilde{A}_{\mu}^{a}(p) \tilde{B}_{\nu}^{b}(s, q)\right\rangle=\left\langle\tilde{B}_{\mu}^{a}(0, p) \tilde{B}_{\nu}^{b}(s, q)\right\rangle
$$

as well as the two-point function of the gauge field at flow time zero.

Since all three propagators are given by the same analytic expression, the same graphical symbol

$$
\sim_{\vec{p}}^{t, \mu, a} \sim^{s, v, b}=\delta^{a b} g_{0}^{2} \tilde{D}_{t+s}(p)_{\mu \nu}
$$


may be used for them. Note that the contraction of the one-point vertices always has the effect of converting the terminal flow lines to gauge-field lines. If one starts from the flow-line diagram
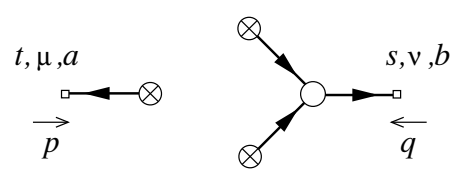

instead of the diagram (3.5), for example, the leading-order contribution is obtained by substituting the tree-level diagram for the correlation function of the gauge fields at the three one-point vertices. This leads to a diagram

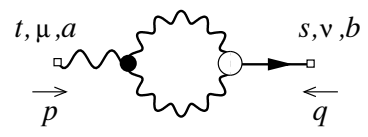

that has an ordinary three-point vertex (filled circle) and a flow vertex. Two of the lines attached to the latter are gauge-field lines instead of flow lines, but the expression for the vertex is the same as before.

\subsection{Feynman rules in $D+1$ dimensions}

The flow time will now be interpreted as an additional space-time coordinate. Since only non-negative times are considered, the $D+1$ dimensional space is a half-space with a $D$ dimensional boundary at flow time zero. The $\mathrm{SU}(N)$ gauge theory lives at the boundary, while the field generated by the gradient flow extends to the extra dimension.

From this point of view, the ordinary and the flow vertices represent boundary and bulk interaction terms, respectively, while the propagation of the fields in $D+1$ dimensions is described by the gauge-field propagator (3.9) and the flow propagator (2.16). The ghostfield propagator

$$
\left\langle\tilde{c}^{a}(p) \tilde{\bar{c}}^{b}(q)\right\rangle=(2 \pi)^{D} \delta(p+q) \delta^{a b} g_{0}^{2} \tilde{D}(p)+\mathrm{O}\left(g_{0}^{4}\right), \quad \tilde{D}(p)=\frac{1}{p^{2}},
$$

on the other hand, is defined at flow time zero only. Graphically it is represented through a dotted line,

$$
\stackrel{\substack{a \\ \cdots \cdots \cdots \cdots \cdots}}{b}=\delta^{a b} g_{0}^{2} \tilde{D}(p),
$$

where the arrow is drawn in the direction from $\bar{c}$ to $c$.

It should be quite clear at this point that the $n$-point functions of the field generated by the gradient flow are given by Feynman diagrams in $D+1$ dimensions with the graphical elements listed in the caption of figure 2. Some special features of the Feynman rules are worth pointing out:

(a) Each flow vertex has exactly one outward-directed flow line corresponding to the first momentum-index combination in eq. (2.13). The other lines attached to these vertices may be gauge-field lines or ingoing flow lines. 

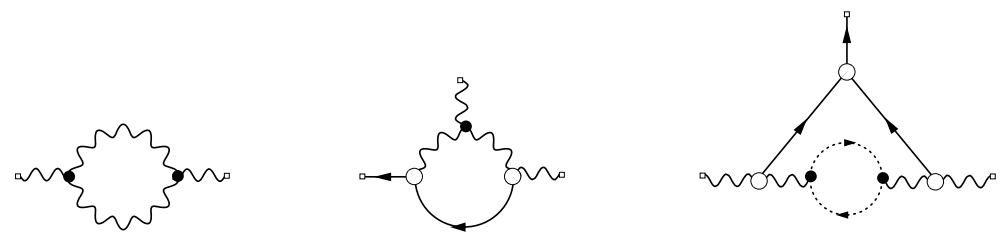

Figure 2. Examples of diagrams contributing to the two- and three-point functions of the timedependent gauge field. All diagrams are built from flow propagators (directed solid lines), gaugefield propagators (wiggly lines), ghost-field propagators (directed dotted lines), flow vertices (open circles) and ordinary vertices at flow time zero (filled circles). The little square at the end of the external lines indicates that they are not amputated.

(b) Flow lines must start at a flow vertex and are either external or end at another flow vertex. The gauge-field lines, on the other hand, can start and end at both the flow vertices and the ordinary vertices.

(c) The flow vertices are inserted at some flow time which is integrated from zero to infinity. All other vertices are at flow time zero. The flow times on which the propagators depend are the ones at the endpoints of the corresponding lines.

(d) Diagrams with closed flow-line loops are set to zero. This rule derives from the fact that flow-line diagrams are tree diagrams and that the contraction of the gauge fields at the one-point vertices never leads to new flow lines.

\section{Field theory $D+1$ dimensions}

The Feynman rules for the correlation functions of the time-dependent gauge field obtained in the previous section are those of a local field theory in $D+1$ dimensions [4]. It is possible to show this through somewhat formal functional-integral manipulations, but one can also adopt a purely algebraic point of view, where the action in $D+1$ dimensions merely serves as a generating function for the Feynman rules.

\subsection{Action}

Except for the boundary condition (2.3), the field $B_{\mu}(t, x)$ will now be considered to be an independent field. One also needs to introduce a Lagrange-multiplier field $L_{\mu}(t, x)=$ $L_{\mu}^{a}(t, x) T^{a}$ with purely imaginary components. The action of the theory in $D+1$ dimensions is then given by [4]

$$
\begin{aligned}
S_{\mathrm{tot}} & =S+S_{\mathrm{gf}}+S_{c \bar{c}}+S_{\mathrm{fl}}, \\
S_{\mathrm{fl}} & =-2 \int_{0}^{\infty} \mathrm{d} t \int \mathrm{d}^{D} x \operatorname{tr}\left\{L_{\mu}(t, x)\left(\partial_{t} B_{\mu}-D_{\nu} G_{\nu \mu}-\alpha_{0} D_{\mu} \partial_{\nu} B_{\nu}\right)(t, x)\right\} .
\end{aligned}
$$

In this framework, the flow equation (2.4) coincides with the field equation obtained by varying the action with respect to the Lagrange-multiplier field. Note that the latter is not required to satisfy any particular boundary conditions. 


\subsection{Propagators}

The quadratic part of the action is the sum of

$$
\int_{0}^{\infty} \mathrm{d} t \int \mathrm{d}^{D} x L_{\mu}^{a}(t, x)\left(\partial_{t} B_{\mu}^{a}-\partial_{\nu} \partial_{\nu} B_{\mu}^{a}-\left(\alpha_{0}-1\right) \partial_{\mu} \partial_{\nu} B_{\nu}^{a}\right)(t, x)
$$

and the quadratic part of the action in $D$ dimensions. The bulk fields $B_{\mu}$ and $L_{\mu}$ thus couple to each other only, but there is also an implicit coupling to the fundamental gauge field through the boundary condition (2.3). This complication can easily be overcome by substituting

$$
B_{\mu}(t, x)=\int \mathrm{d}^{D} y K_{t}(x-y)_{\mu \nu} A_{\nu}(y)+b_{\mu}(t, x) .
$$

The field $b_{\mu}$ then satisfies homogenous boundary conditions, while the first term in eq. (4.4) solves the linearized flow equation and thus drops out in the action (4.3).

It follows from these remarks that the propagators of the fundamental gauge field and the ghost fields coincide with the expressions given in the previous section. All other two-point functions vanish except for

$$
\left.\left\langle b_{\mu}^{a}(t, x) L_{\nu}^{b}(s, y)\right\rangle\right|_{\text {leading order }}=\delta^{a b} H(t, x ; s, y)_{\mu \nu},
$$

which is determined by the field equation

$$
\left\{\delta_{\mu \rho} \partial_{t}-\delta_{\mu \rho} \partial_{\sigma} \partial_{\sigma}-\left(\alpha_{0}-1\right) \partial_{\mu} \partial_{\rho}\right\} H(t, x ; s, y)_{\rho \nu}=\delta_{\mu \nu} \delta(t-s) \delta(x-y)
$$

and the boundary condition $\left.H_{\mu \nu}(t, x ; s, y)\right|_{t=0, s>0}=0$. The unique solution of these equations is

$$
H(t, x ; s, y)_{\mu \nu}=\theta(t-s) K_{t-s}(x-y)_{\mu \nu}
$$

and the $b L$ propagator is thus seen to coincide with the flow propagator.

The two-point functions involving the $B$ field may finally be calculated by combining eq. (4.4) with the results obtained so far. Since the $A L$ and the $b b$ propagators vanish, one quickly finds that the $B L$ propagator is equal to the flow propagator and that the $B B$ and the $A B$ propagators are equal to the expressions given in the previous section.

\subsection{Vertices}

The theory described by the action (4.1) has the vertices of the theory in $D$ dimensions plus those deriving from the bulk action $S_{\mathrm{fl}}$. Recalling eqs. (2.6) and (2.13), it is straightforward to show that the $L B^{2}$ and $L B^{3}$ vertices generated by the interaction part of the latter,

$$
\left.S_{\mathrm{ff}}\right|_{\text {interaction }}=2 \int_{0}^{\infty} \mathrm{d} t \int \mathrm{d}^{D} x \operatorname{tr}\left\{L_{\mu}(t, x) R_{\mu}(t, x)\right\},
$$

coincide with the vertices $X^{(2,0)}$ and $X^{(3,0)}$.

All vertices and propagators of the Feynman rules of the previous sections are thus recovered. A somewhat unusual feature of these rules is the presence of a field (the Lagrange multiplier) that propagates only through its mixing with the other fields. The algebraic structure of the Feynman rules is however entirely standard and does not involve any prescriptions apart from the ones deriving from the expansion of the action in powers of the fields. 


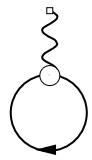

1

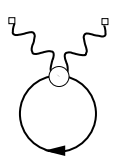

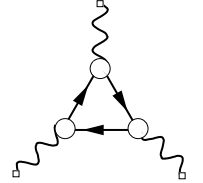

Figure 3. Examples of diagrams with flow-line loops. All such diagrams vanish as a consequence of the retarded nature of the flow propagator and the rules of dimensional regularization [4].

\subsection{Flow lines and flow-line loops}

Flow lines represent the $B L$ propagator. They can start and end at the flow vertices but are never attached to an ordinary vertex. Outward- and inward-directed external flow lines are external $B$ and $L$ lines, respectively.

Diagrams with closed flow lines comply with these rules, but should be absent if a complete matching with the Feynman rules of the previous section is to be achieved (cf. point (d) at the end of section 3). Such diagrams are in fact equal to zero [4]. The diagrams 1 and 2 in figure 3, for example, vanish because dimensional regularization sets the momentum integral to zero. If there are two or more vertices in the loop, as in diagram 3 , the time integrations vanish, because the flow propagator is retarded and thus forces the flow times at the vertices to be squeezed to a range of measure zero (singularities in the time coordinates are excluded in view of the regularization of the momentum integral).

If a lattice regularization is used, the vanishing of diagram 2 may not be guaranteed, but one can always include a ghost field in the action that cancels the flow-line loops algebraically, i.e. at the level of the Feynman integrands [3]. With dimensional regularization, however, this device is not needed and is therefore omitted here.

\section{Sample calculation at one-loop order}

For illustration, the divergent parts of the one-loop diagrams that contribute to the gaugefield two-point functions are worked out in this section. The parameter and field renormalization required at flow time zero is then seen to cancel the singularities at all flow times.

\subsection{Computation of self-energy diagrams}

There are only few self-energy diagrams at one-loop order (see figure 4). The diagrams $1-3$ coincide with the ones contributing to the two-point function of the gauge field at flow time zero. We therefore merely quote the known result

$$
\left.\Gamma_{A A}^{(1)}(p)_{\mu \nu}^{a b}\right|_{\mathrm{pole}}=\delta^{a b}\left(\delta_{\mu \nu} p^{2}-p_{\mu} p_{\nu}\right) \frac{N}{16 \pi^{2} \epsilon}\left(\frac{13}{6}-\frac{1}{2 \lambda_{0}}\right)
$$

for the sum of their singular parts.

In the case of diagram 4, the Feynman integrand is a linear combination of terms of the form

$$
\delta^{a b} \frac{Q(k, p)_{\mu \nu}}{\left\{k^{2}(k+p)^{2}\right\}^{2}} \mathrm{e}^{-t\left\{u k^{2}+v(k+p)^{2}\right\}}, \quad u, v \in\left\{1, \alpha_{0}\right\}
$$




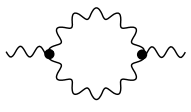

1

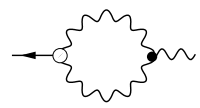

4

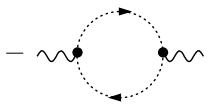

2

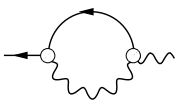

5

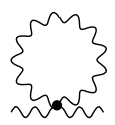

3

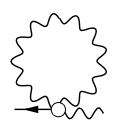

6

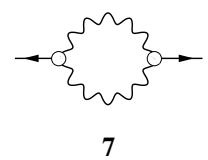

7

Figure 4. One-loop diagrams contributing to the $A A(1-3), L A(4), L B(5,6)$ and $L L(7)$ vertex functions. The time-momentum-index combinations at the external legs on the left and right are $(t, p, \mu, a)$ and $(s, q, \nu, b)$, respectively (the flow times are absent in the case of the $A$ legs).

where $k$ is the loop momentum and $Q(k, p)_{\mu \nu}$ a homogeneous polynomial in $k$ and $p$ of degree 6 (the momentum-conservation factor $(2 \pi)^{D} \delta(p+q)$ is now always suppressed). This diagram is finite at all $t>0$ and in any dimension $D$, but eventually it will be multiplied by the external propagators and must then be integrated over $t$ from 0 to infinity. The behaviour of the integral small $t$ thus matters and can give rise to singularities at $D=4$.

If $f(t)$ is any smooth test function (an external propagator, for example), the time integral

$$
I(k, p)=\int_{0}^{\infty} \mathrm{d} t f(t) \mathrm{e}^{-t\left\{u k^{2}+v(k+p)^{2}\right\}}
$$

can be worked out in an asymptotic series at large $k$, the leading term being

$$
I(k, p)=\frac{f(0)}{(u+v) k^{2}}+\mathrm{O}\left(k^{-3}\right) .
$$

The integration over the flow time $t$ thus improves the degree of divergence of the momentum integral. In particular, the first term in the decomposition

$$
I(k, p)=\left\{I(k, p)-\frac{f(0)}{(u+v)(k+r)^{2}}\right\}+\frac{f(0)}{(u+v)(k+r)^{2}}
$$

(where $r$ is an arbitrary external momentum) makes the integral convergent at $D=4$. One is then left with a logarithmic divergence and therefore a pole singularity equal to the one of the ordinary Feynman integral

$$
\delta^{a b} \delta(t) \frac{1}{u+v} \int_{k} \frac{Q(k, 0)_{\mu \nu}}{\left(k^{2}\right)^{4}(k+r)^{2}},
$$

which is easily evaluated using standard techniques.

Proceeding in this way, one obtains

$$
\begin{gathered}
\left.\Gamma_{L A}^{(1)}(t, p)_{\mu \nu}^{a b}\right|_{\text {pole }}=g_{0}^{2} \delta^{a b} \delta(t) \delta_{\mu \nu} \frac{N}{16 \pi^{2} \epsilon}\left(\frac{3}{4}+\frac{3}{4 \lambda_{0}}\right), \\
\left.\Gamma_{L B}^{(1)}(t, s, p)_{\mu \nu}^{a b}\right|_{\text {pole }}=g_{0}^{2} \delta^{a b} \delta(t) \delta(s) \delta_{\mu \nu} \frac{N}{16 \pi^{2} \epsilon}\left(-\frac{1}{2 \lambda_{0}}\right),
\end{gathered}
$$

for the divergent parts of the $L A$ and $L B$ vertex functions. The diagram 7 and thus the $L L$ vertex function are finite at $D=4$. 


\subsection{Renormalization}

The bare coupling and gauge-fixing parameter are related to the renormalized parameters $g$ and $\lambda$ through

$$
g_{0}^{2}=\mu^{2 \epsilon} g^{2} Z, \quad \lambda_{0}=\lambda Z_{3}^{-1},
$$

where $\mu$ is the normalization mass. To one-loop order, the renormalization constants $Z$ and $Z_{3}$ are given by

$$
\begin{aligned}
Z & =1-\frac{b_{0}}{\epsilon} g^{2}+\mathrm{O}\left(g^{4}\right), & b_{0} & =\frac{N}{16 \pi^{2}} \frac{11}{3}, \\
Z_{3} & =1+\frac{c_{0}}{\epsilon} g^{2}+\mathrm{O}\left(g^{4}\right), & c_{0} & =\frac{N}{16 \pi^{2}}\left(\frac{13}{6}-\frac{1}{2 \lambda}\right),
\end{aligned}
$$

up to scheme-dependent finite terms.

The renormalization of the fundamental gauge field,

$$
A_{\mu}^{a}=Z^{1 / 2} Z_{3}^{1 / 2}\left(A_{\mathrm{R}}\right)_{\mu}^{a},
$$

involves both renormalization constants as a result of the unconventional normalization conventions adopted in this paper. For reasons explained in section 7, the ghost fields are renormalized asymmetrically according to

$$
c^{a}=\tilde{Z}_{3} Z^{1 / 2} Z_{3}^{1 / 2}\left(c_{\mathrm{R}}\right)^{a}, \quad \bar{c}^{a}=Z^{1 / 2} Z_{3}^{-1 / 2}\left(\bar{c}_{\mathrm{R}}\right)^{a},
$$

where $\tilde{Z}_{3}$ is the usual ghost renormalization constant. Note that eq. (5.13) is equivalent to the standard renormalization prescription for the correlation functions at flow time zero, because the fields $c$ and $\bar{c}$ always occur in pairs and only the product of their renormalization factors matters.

\subsection{Do the bulk fields require renormalization?}

To one-loop order of perturbation theory, the question may be answered by explicitly calculating the divergent parts (if any) of bulk-field correlation functions. We first consider the two-point function of the $B$ field and define its Lorentz-invariant parts $\mathcal{A}$ and $\mathcal{B}$ through

$$
\begin{aligned}
\left\langle\tilde{B}_{\mu}^{a}(t, p) \tilde{B}_{\nu}^{b}(s, q)\right\rangle= & (2 \pi)^{D} \delta(p+q) \frac{\delta^{a b}}{\left(p^{2}\right)^{2}} \\
& \times\left\{\left(\delta_{\mu \nu} p^{2}-p_{\mu} p_{\nu}\right) \mathcal{A}\left(t, s, p^{2}\right)+p_{\mu} p_{\nu} \mathcal{B}\left(t, s, p^{2}\right)\right\} .
\end{aligned}
$$

All self-energy diagrams drawn in figure 4 contribute to $\mathcal{A}$ and $\mathcal{B}$. The diagrams $4-6$ actually make two contributions, because their external legs are different.

In the renormalized perturbation expansion

$$
\mathcal{X}=\mu^{2 \epsilon} \sum_{l=0}^{\infty} g^{2 l+2} \mathcal{X}^{(l)}, \quad \mathcal{X}=\mathcal{A} \text { or } \mathcal{B},
$$

the leading-order coefficients are

$$
\mathcal{A}^{(0)}=\mathrm{e}^{-(t+s) p^{2}}, \quad \mathcal{B}^{(0)}=\lambda^{-1} \mathrm{e}^{-\alpha_{0}(t+s) p^{2}} .
$$


At the next order, the residues of the poles that derive from the renormalization of the coupling and the gauge-fixing parameter are thus given by

$$
\begin{aligned}
& \left.\operatorname{res}\left\{\mathcal{A}^{(1)}\right\}\right|_{Z \text { factors }}=-b_{0} \mathcal{A}^{(0)}, \\
& \left.\operatorname{res}\left\{\mathcal{B}^{(1)}\right\}\right|_{Z \text { factors }}=\left(c_{0}-b_{0}\right) \mathcal{B}^{(0)} .
\end{aligned}
$$

Recalling the results obtained in subsection 5.1, it is now straightforward to verify that these poles are canceled by the divergent parts of the one-loop diagrams. Up to this order of perturbation theory, the two-point function of the time-dependent gauge field is thus finite and does not require further renormalization.

In the case of the $B L, B A_{\mathrm{R}}, L A_{\mathrm{R}}$ and $L L$ correlation functions, the cancellation of the singularities at $D=4$ can be shown in the same way. There is actually little to prove in the last two instances, because these two-point functions vanish to all orders of perturbation theory (there are no diagrams with ingoing and no outgoing flow lines). All calculations reported in this section thus support the conjecture that the $B$ and the $L$ field do not need to be renormalized.

\section{BRS symmetry}

We now proceed with the general discussion that will lead to the proof of finiteness of the correlation functions of the bulk fields to all orders in the gauge coupling. As a first step, the BRS symmetry of the theory in $D+1$ dimensions is reviewed in this section.

The gauge fixing discussed in section 3.1 follows the standard procedure and therefore leads to a theory with a BRS symmetry. This symmetry acts on the boundary fields in the usual way, but the transformation of the bulk fields requires the solution of the diffusion equation (3.2) and consequently tends to be non-local. In their work on the renormalization of the Langevin equation, Zinn-Justin and Zwanziger [4] however showed that the locality of the transformation can be restored by introducing additional ghost fields.

\subsection{BRS transformation of the boundary fields}

The BRS variation of the unrenormalized fields $A_{\mu}, \bar{c}$ and $c$ is defined by $[5,6]$

$$
\begin{aligned}
\delta A_{\mu} & =D_{\mu} c, \\
\delta c & =-c^{2}, \\
\delta \bar{c} & =\lambda_{0} \partial_{\mu} A_{\mu} .
\end{aligned}
$$

Note that the components $c^{a}$ and $\bar{c}^{a}$ of the ghost fields and the operator $\delta$ anti-commute with one another. The product on the right of eq. (6.2), for example, is given by

$$
c^{2}=c^{a} c^{b} T^{a} T^{b}=\frac{1}{2} c^{a} c^{b} f^{a b c} T^{c},
$$

and the Leibniz rule for the operator $\delta$ must take its anti-commuting character into account. The action $S+S_{\mathrm{gf}}+S_{c \bar{c}}$ and the measure in the functional integral of the theory in $D$ dimensions are then easily shown to be BRS invariant. 


\subsection{Bulk ghost fields}

The additional ghost fields $d(t, x)$ and $\bar{d}(t, x)$ mentioned above live in $D+1$ dimensions but are otherwise of the same kind as the Faddeev-Popov ghosts. Their action is

$$
S_{d \bar{d}}=-2 \int_{0}^{\infty} \mathrm{d} t \int \mathrm{d}^{D} x \operatorname{tr}\left\{\bar{d}(t, x)\left(\partial_{t} d-\alpha_{0} D_{\mu} \partial_{\mu} d\right)(t, x)\right\}
$$

and the $d$ field is required to satisfy the boundary condition

$$
\left.d\right|_{t=0}=c .
$$

No boundary condition is imposed on the $\bar{d}$ field, which, in many respects, plays a rôle similar to the Lagrange-multiplier field $L_{\mu}$ in the case of the gauge field.

The propagator of the ghost fields,

$$
\begin{aligned}
\left\langle\tilde{d}^{a}(t, p) \tilde{\tilde{d}}^{b}(s, q)\right\rangle & =(2 \pi)^{D} \delta(p+q) \delta^{a b} \theta(t-s) \tilde{K}_{t-s}(p)+\mathrm{O}\left(g_{0}^{2}\right), \\
\tilde{K}_{t}(p) & =\mathrm{e}^{-\alpha_{0} t p^{2}}
\end{aligned}
$$

can be worked out following the steps taken in subsection 4.2. Graphically the propagator is represented by a dashed line,

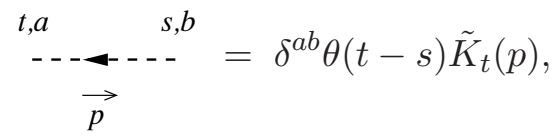

where the arrow is drawn in the direction from $\bar{d}$ to $d$. There is a mixed propagator,

$$
\begin{aligned}
\left\langle\tilde{d}^{a}(t, p) \tilde{\bar{c}}^{b}(q)\right\rangle & =(2 \pi)^{D} \delta(p+q) \delta^{a b} g_{0}^{2} \tilde{D}_{t}(p)+\mathrm{O}\left(g_{0}^{4}\right), \\
\tilde{D}_{t}(p) & =\frac{1}{p^{2}} \mathrm{e}^{-\alpha_{0} t p^{2}},
\end{aligned}
$$

as well, which coincides with the $c \bar{c}$ propagator at $t=0$ and is therefore represented by the same graphical symbol (a directed dotted line). The $c \bar{d}$ two-point function, on the other hand, vanishes to all orders.

The action

$$
\begin{aligned}
\left.S_{d \bar{d}}\right|_{\text {interaction }}= & -\int_{0}^{\infty} \mathrm{d} t \int_{p, q, r}(2 \pi)^{D} \delta(p+q+r) \\
& \times X^{(1,1)}(p, q, r)_{\mu}^{a b c} \tilde{B}_{\mu}^{a}(t,-p) \tilde{\bar{d}}(t,-q)^{b} \tilde{d}(t,-r)^{c},
\end{aligned}
$$

also gives rise to a new flow vertex $X^{(1,1)}$. In the Feynman diagrams, it is represented by an open circle as the other flow vertices. The explicit expression for the vertex is given in appendix B.

As in the case of the flow-line loops discussed in subsection 4.4, it is possible to show that diagrams with $d \bar{d}$ loops vanish. Note that ghost loops with mixed propagators do not exist, because there is no $c \bar{d}$ propagator and the vertices only couple $c$ to $\bar{c}$ or $d$ to $\bar{d}$. The only non-zero ghost loops are thus the usual ones of the theory at flow time zero. 


\subsection{BRS variation of the fields in the bulk}

The BRS symmetry acts on the bulk fields according to [4]

$$
\begin{aligned}
\delta B_{\mu} & =D_{\mu} d, \\
\delta L_{\mu} & =\left[L_{\mu}, d\right], \\
\delta d & =-d^{2}, \\
\delta \bar{d} & =D_{\mu} L_{\mu}-\{d, \bar{d}\} .
\end{aligned}
$$

Note that $\delta B_{\mu}=\delta A_{\mu}$ and $\delta d=\delta c$ at the boundary, as must be the case in view of the boundary conditions (2.3) and (6.6).

In order to show that the BRS variation of the bulk action $S_{\mathrm{fl}}+S_{d \bar{d}}$ vanishes, it is helpful to introduce the fields

$$
\begin{aligned}
E_{\mu} & =\partial_{t} B_{\mu}-D_{\nu} G_{\nu \mu}-\alpha_{0} D_{\mu} \partial_{\nu} B_{\nu} \\
e & =\partial_{t} d-\alpha_{0} D_{\mu} \partial_{\mu} d .
\end{aligned}
$$

After some algebra, one finds that

$$
\begin{aligned}
\delta E_{\mu} & =\left[E_{\mu}, d\right]+D_{\mu} e, \\
\delta e & =-\{e, d\}
\end{aligned}
$$

and the invariance of the bulk action is then easily established.

\subsection{Examples of BRS identities}

Since the functional integration measure is also invariant, it follows that $\langle\delta \mathcal{O}\rangle=0$ for any product $\mathcal{O}$ of the basic fields. This leads to identities such as

$$
\begin{aligned}
\lambda_{0}\left\langle\partial_{\mu} A_{\mu}^{a}(x) \partial_{\nu} A_{\nu}^{b}(y)\right\rangle & =g_{0}^{2} \delta^{a b} \delta(x-y), \\
\lambda_{0}\left\langle B_{\mu}^{a}(t, x) \partial_{\nu} A_{\nu}^{b}(y)\right\rangle & =-\left\langle\left(D_{\mu} d\right)^{a}(t, x) \bar{c}^{b}(y)\right\rangle, \\
\lambda_{0}\left\langle B_{\mu}^{a}(t, x) \partial_{\nu} A_{\nu}^{b}(y) \partial_{\rho} A_{\rho}^{c}(z)\right\rangle & =-\left\langle\left(D_{\mu} d\right)^{a}(t, x) \bar{c}^{b}(y) \partial_{\rho} A_{\rho}^{c}(z)\right\rangle,
\end{aligned}
$$

the first of them being the familiar gauge Ward identity in the theory in $D$ dimensions, which determines the longitudinal part of the gauge-field two-point function.

It is instructive to check eqs. (6.22) and (6.23) at tree level of perturbation theory. The first equation relates the longitudinal part of the mixed gauge-field propagator to the mixed ghost propagator, an identity that is immediate from the expressions for the propagators. The other equation involves the 3-point vertices (see figure 5) and a non-trivial cancellation among various contributions (appendix $\mathrm{C}$ ). 


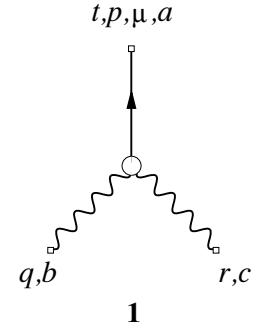

1

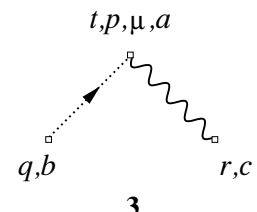

3
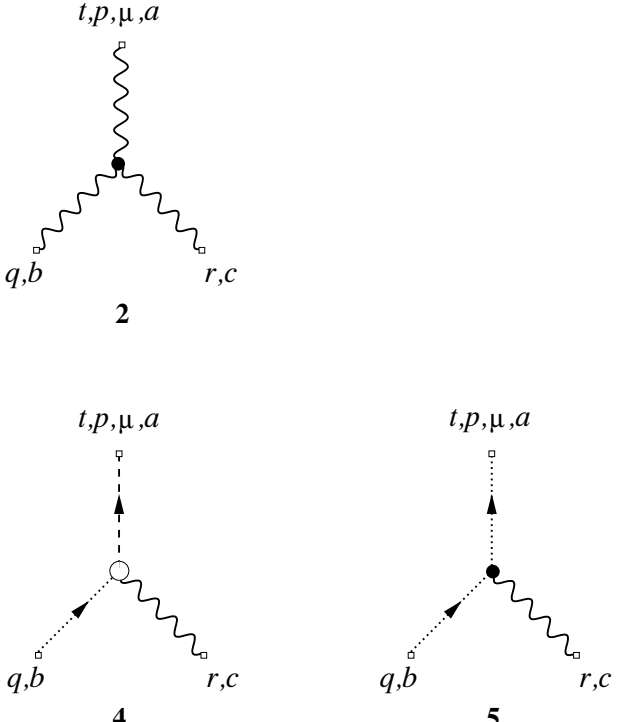

5

Figure 5. Tree diagrams contributing to the correlation function on the left of eq. (6.23) (diagrams 1 and 2) and to the one on the right of the equation (diagrams 3,4 and 5). The momenta are ingoing and satisfy $p+q+r=0$. In diagram 3 , the momentum $p$ flows into a vertex representing the insertion of the field $\left[B_{\mu}, d\right]$, while in the case of the diagrams 4 and 5 the external lines with momentum $p$ are multiplied by $i p_{\mu}$ (thus representing the field $\partial_{\mu} d$ ).

\section{Finiteness of the renormalized perturbation expansion}

The renormalization constants $Z, Z_{3}$ and $\tilde{Z}_{3}$ are now assumed to be such that the singularities of the correlation functions of the fields $\left(A_{\mathrm{R}}\right)_{\mu}, c_{\mathrm{R}}$ and $\bar{c}_{\mathrm{R}}$ at $D=4$ cancel to all orders of the renormalized coupling. Our aim in this section is to show that the correlation functions involving the bulk fields at positive flow time are then finite too and thus do not require further renormalization.

\subsection{Renormalized perturbation theory}

We first need to reorganize the perturbation expansion in terms of the renormalized parameters and fields. In this form, the expansion is generated by an action $S_{0}+\Delta S$ of the renormalized fields, which includes the counterterms $\Delta S$ required to cancel the poles of the Feynman diagrams at $D=4$.

Explicitly $S_{0}$ and $\Delta S$ are obtained by expressing the bare parameters and fields in the total action

$$
S_{\mathrm{tot}}=S+S_{\mathrm{gf}}+S_{c \bar{c}}+S_{\mathrm{fl}}+S_{d \bar{d}}
$$

through the renormalized ones and by setting

$$
\begin{gathered}
S_{0}=\left.S_{\mathrm{tot}}\right|_{Z=Z_{3}=\tilde{Z}_{3}=1}, \\
\Delta S=S_{\mathrm{tot}}-S_{0}+\Delta S_{\mathrm{bc}},
\end{gathered}
$$


where

$$
\begin{aligned}
\Delta S_{\mathrm{bc}}=2 \int \mathrm{d}^{D} x \operatorname{tr}\{( & \left.Z^{1 / 2} Z_{3}^{1 / 2}-1\right) L_{\mu}(0, x)\left(A_{\mathrm{R}}\right)_{\mu}(x) \\
& \left.+\left(\tilde{Z}_{3} Z^{1 / 2} Z_{3}^{1 / 2}-1\right) \bar{d}(0, x) c_{\mathrm{R}}(x)\right\} .
\end{aligned}
$$

The boundary conditions are then

$$
\left.B_{\mu}\right|_{t=0}=\left(A_{\mathrm{R}}\right)_{\mu},\left.\quad d\right|_{t=0}=c_{\mathrm{R}},
$$

and the Feynman rules derived from the action $S_{0}$ thus coincide with those discussed in section 3 (apart from the fact that the bare parameters $g_{0}$ and $\lambda_{0}$ are replaced by $\mu^{\epsilon} g$ and $\lambda$, respectively).

Note that the boundary conditions (7.5) differ from the ones imposed on the bare fields (a product of renormalization factors is missing). The counterterm $\Delta S_{\mathrm{bc}}$ must be included in the action of the renormalized fields to correct for this. It amounts to adding two-point vertices to the Feynman rules, whose effect on the gauge-field and ghost propagators is equivalent to a change of the boundary conditions by the missing renormalization factors.

\subsection{Absence of bulk counterterms}

In the renormalized perturbation expansion, singularities at $D=4$ (if any) appear for the first time at some loop order $l \geq 1$. Since the interactions are local, and since all propagators are tempered distributions in position space with singularities only at coinciding arguments, the divergent parts of the correlation functions at this loop order are expected to be such that they can be canceled by local counterterms. Similarly to the case of an ordinary field theory on a half-space studied by Symanzik [7], the counterterms can be localized either in the bulk or at the boundary of the half-space.

In the theory considered here, bulk counterterms can be excluded from the outset. In order to show this, first note that the correlation functions of the bulk fields $B_{\mu}, L_{\mu}, d$ and $\bar{d}$ are, at large flow times, given by Feynman diagrams built from flow lines and flow vertices only. All diagrams of this kind are directed trees or products such trees, each tree ending at one of the $B$ and $d$ fields in the correlation function considered. Moreover, the flow lines at the other ends of the trees must start from the $L$ and $\bar{d}$ fields in the correlation function.

Since there are no loop diagrams, the correlation functions of the bulk fields are nonsingular at large flow times and do not require renormalization. Divergent bulk counterterms are therefore excluded. Note, incidentally, that correlation functions of local fields composed from $B$ and $d$ fields are finite too, because no loop diagrams are generated when the arguments of some of these fields coalesce. The field $D_{\mu} d$ that appears in the BRS identity (6.23), for example, is of this kind and consequently does not need to be renormalized.

\subsection{Boundary counterterms}

The discussion in the previous subsection shows that the structure of any divergent parts of the correlation functions must correspond to the insertion of a counterterm localized at flow time zero. Since the correlation functions of the renormalized fields $\left(A_{\mathrm{R}}\right)_{\mu}, c_{\mathrm{R}}$ and $\bar{c}_{\mathrm{R}}$ are finite to all orders, divergences can only arise from diagrams with at least one flow vertex and thus at least one such vertex with an external flow line. The possible counterterms are therefore proportional to $L_{\mu}$ or $\bar{d}$. 
Since the gauge coupling is dimensionless, the counterterm may not involve composite fields of dimension larger than 4 (such terms would be irrelevant). Moreover, Lorentz symmetry, global gauge invariance and the ghost number conservation must be respected. Since $L_{\mu}$ and $\bar{d}$ have dimension 3 and all other fields dimension 1, it follows that the possible counterterms at $l$-loop order are of the form

$$
2 g^{2 l} \int \mathrm{d}^{D} x \operatorname{tr}\left\{z_{1} L_{\mu}(0, x)\left(A_{\mathrm{R}}\right)_{\mu}(x)+z_{2} \bar{d}(0, x) c_{\mathrm{R}}(x)\right\}
$$

with some (singular) coefficients $z_{1}$ and $z_{2}$. An $L B$ and a $\bar{d} d$ term should in principle be included here, but in view of the boundary conditions (7.5), these terms are not independent and their inclusion would be equivalent to a change of the coefficients $z_{1}$ and $z_{2}$.

\subsection{Consequences of the BRS symmetry}

We now show that the boundary counterterm (7.6) is excluded by the BRS symmetry of the theory. In terms of the renormalized fields and parameters, the BRS identity (6.23) reads

$$
\lambda\left\langle B_{\mu}^{a}(t, x) \partial_{\nu}\left(A_{\mathrm{R}}\right)_{\nu}^{b}(y) \partial_{\rho}\left(A_{\mathrm{R}}\right)_{\rho}^{c}(z)\right\rangle=-\left\langle\left(D_{\mu} d\right)^{a}(t, x)\left(\bar{c}_{\mathrm{R}}\right)^{b}(y) \partial_{\rho}\left(A_{\mathrm{R}}\right)_{\rho}^{c}(z)\right\rangle .
$$

The unusual asymmetric renormalization (5.13) of the ghost fields $c$ and $\bar{c}$ was chosen to ensure that the renormalization factors drop out in this equation. From the point of view of the $\mathrm{SU}(N)$ gauge theory, the standard and the asymmetric renormalization of the ghost fields are equivalent, but the situation is different in the theory in $D+1$ dimensions, because only $c$ couples to the bulk fields.

Equation (7.7) holds as long as no extra counterterms need to be added and thus up to loop order $l$ inclusive. Note that all fields in the correlation functions in this equation, including the composite field $D_{\mu} d$, are renormalized fields that cannot be additionally renormalized (cf. subsection 7.2). If the correlation functions are singular at $l$-loop order, one must therefore be able cancel the singularities by a counterterm of the form (7.6).

The contribution of the counterterm (7.6) to the correlation functions is obtained by inserting the corresponding two-point vertices in the tree diagrams in figure 5 . The insertions have the effect of multiplying the values of the diagrams $1-5$ by $g^{2 l}$ times

$$
2 z_{1}, z_{1}, z_{1}+z_{2}, z_{1}+z_{2} \text { and } z_{2}
$$

respectively. However, recalling the values of the diagrams quoted in appendix $\mathrm{C}$, the sum of the diagrams weighted by the factors (7.8) turns out to violate the BRS identity (7.7) unless $z_{1}=z_{2}=0$.

The correlation functions in eq. (7.7) must therefore be finite at $l$-loop order and all other (renormalized) correlation functions must be non-singular too, because the addition to the action of a counterterm of the form (7.6) is excluded. We have thus shown that the theory in $D+1$ dimensions does not require further renormalization. 


\section{Miscellaneous remarks}

\subsection{Behaviour of the gauge field near flow time zero}

In the regularized theory, the time-dependent field $B_{\mu}(t, x)$ satisfies the boundary condition (2.3) and its correlation functions thus converge to those of the bare gauge field $A_{\mu}(x)$ when $t$ goes to zero. However, since the latter requires renormalization by a divergent constant, the correlation functions tend to become singular at $t=0$ after renormalization and removal of the regularization. Their asymptotic behaviour for $t \rightarrow 0$ is then described by an expansion

$$
B_{\mu}(t, x)=c_{B}(t)\left(A_{\mathrm{R}}\right)_{\mu}(x)+\mathrm{O}(t)
$$

in local renormalized fields with singular coefficients [7]. At one-loop order of perturbation theory, for example, the coefficient $c_{B}(t)$ is found to diverge logarithmically at $t=0$.

It is straightforward to show that the renormalization group equation

$$
\left\{\mu \frac{\partial}{\partial \mu}+\beta \frac{\partial}{\partial g}-2 \gamma \lambda \frac{\partial}{\partial \lambda}+\gamma+\beta / g\right\} c_{B}(t)=\mathrm{O}(t)
$$

holds, where

$$
\beta=-b_{0} g^{3}+\mathrm{O}\left(g^{5}\right), \quad \gamma=c_{0} g^{2}+\mathrm{O}\left(g^{4}\right),
$$

are the beta function and the anomalous dimension of the gauge field. In the Landau gauge, for example, the equation can be easily integrated and one finds that

$$
B_{\mu}(t, x) \underset{t \rightarrow 0}{\sim}\left(2 b_{0} \bar{g}(q)^{2}\right)^{1 / 2-c_{0} / 2 b_{0}} R(g)\left(A_{\mathrm{R}}\right)_{\mu}(x),
$$

$\bar{g}(q)$ being the running coupling at momentum $q=(8 t)^{-1 / 2}$ and $R(g)$ the factor that relates the renormalized to the renormalization-group-invariant gauge field.

Equation (8.4) is a remnant of the boundary condition satisfied by the gauge field in the regularized theory. It shows that the field generated by the flow equation is connected to the fundamental field in a universal manner, i.e. there is no room for finite renormalizations here, the reason being that any such renormalization would violate the BRS symmetry.

\subsection{Gauge-invariant composite fields}

Wilson loops and gauge-invariant local fields are independent of the parameter $\alpha_{0}$ in the flow equation and do not require renormalization at positive flow time. At small flow times, their asymptotic behaviour is determined by the scaling properties of the corresponding renormalized fields in the $\mathrm{SU}(N)$ gauge theory and thus reflects the singular nature of the latter.

For illustration, consider the density

$$
E=\frac{1}{4} G_{\mu \nu}^{a} G_{\mu \nu}^{a}
$$

that has previously been studied in ref. [1]. Since $E$ can mix with the unit field, the dominant term at small flow times,

$$
E(t, x)=\langle E(t, x)\rangle+c_{E}(t)\left\{\frac{1}{4} F_{\mu \nu}^{a} F_{\mu \nu}^{a}\right\}_{\mathrm{R}}(x)+\mathrm{O}(t),
$$


is its expectation value, while the first subleading term is proportional to the renormalized action density of the fundamental field. The associated coefficients, $\langle E(t, x)\rangle$ and $c_{E}(t)$, satisfy a renormalization group equation that allows their exact asymptotic behaviour at small $t$ to be worked out analytically.

\subsection{Theories with matter fields}

In the presence of matter fields, the gradient flow is defined by the same equations as in the pure gauge theory (subsection 2.1). The interesting but rather more complicated case where the matter fields are included in the time evolution is not considered here.

In a renormalizable theory, matter fields are scalar fields of dimension 1 or fermion fields of dimension $3 / 2$. Provided the regularization preserves the gauge symmetry, our argumentation in sections 6 and 7 then carries over literally. In particular, additional boundary counterterms involving the matter fields are excluded by the global symmetries and the requirement that their dimension must be less than or equal to 4 . The finiteness of the correlation functions at positive flow times is therefore again guaranteed.

QCD is a prominent example of a gauge theory that has all the required properties to ensure finiteness, but the same applies to many more theories of interest, including the $\mathrm{SU}(2)$ Higgs model, supersymmetric versions of QCD and technicolour theories.

\subsection{Lattice regularization}

While the Feynman rules are more complicated than with dimensional regularization, the perturbative analysis of the gradient flow on the lattice is not expected to run into fundamental difficulties.

A possible choice of the gradient term in the lattice flow equation is the gradient of the Wilson action [1]. There is however no reason to choose this particular action or to require that it coincides with the gauge action of the theory. As long as the term has the correct form and normalization in the classical continuum limit (and thus at tree-level of perturbation theory), the correlation functions of the time-dependent gauge field will not depend on the exact choices one makes, except for lattice effects vanishing proportionally to a positive power of the lattice spacing $a$.

The continuous Lorentz symmetry is broken on the lattice, but the remaining exact symmetries are sufficient to exclude counterterms that have not already appeared in the continuum theory. We therefore expect that the correlation functions of the timedependent fields do not require renormalization and that their values in the continuum limit are independent of the regularization (up to finite renormalizations of the coupling and the gauge-fixing parameter). Moreover, if the lattice theory is $\mathrm{O}(a)$ improved, the correlation functions are automatically improved too, because there are no candidate $\mathrm{O}(a)$ counterterms with all the required properties.

\section{Conclusions}

The fact that the gauge field generated by the gradient flow does not require renormalization is a consequence of the locality, the symmetries and the parabolic nature of the flow equation. In particular, in the associated field theory in $D+1$ dimensions, bulk counterterms are excluded simply because the evolution of the bulk fields is retarded at large times 
and thus described by tree diagrams. The absence of boundary counterterms other than those needed for the renormalization of the theory at flow time zero is however non-trivial and can only be shown using power-counting and the BRS symmetry.

In presence of matter fields, the situation is essentially unchanged as long as only the gauge field is evolved in time. The finiteness of the correlation functions of the field at positive flow time is therefore still guaranteed. Including all or some of the matter fields in the flow is however an interesting option that remains to be explored.

\section{Acknowledgments}

We thank Jean Zinn-Justin for helpful discussions on the renormalization of the Langevin equation and for encouraging us to proceed directly with the flow equation rather than considering the latter to be a limit of the Langevin equation.

\section{A Notational conventions}

The Lie algebra $\mathfrak{s u}(N)$ of $\mathrm{SU}(N)$ may be identified with the linear space of all anti-hermitian traceless $N \times N$ matrices. With respect to a basis $T^{a}, a=1, \ldots, N^{2}-1$, of such matrices, the elements $X \in \mathfrak{s u}(N)$ are given by $X=X^{a} T^{a}$ with real components $X^{a}$ (repeated group indices are automatically summed over). The structure constants $f^{a b c}$ in the commutator relation

$$
\left[T^{a}, T^{b}\right]=f^{a b c} T^{c}
$$

are real and totally anti-symmetric in the indices if the normalization condition

$$
\operatorname{tr}\left\{T^{a} T^{b}\right\}=-\frac{1}{2} \delta^{a b}
$$

is imposed. Moreover, $f^{a c d} f^{b c d}=N \delta^{a b}$.

Gauge fields in the continuum theory take values in the Lie algebra of the gauge group. Lorentz indices $\mu, \nu, \ldots$ are automatically summed over when they occur in matching pairs. The space-time metric is assumed to be euclidean. In particular, $p^{2}=p_{\mu} p_{\mu}$ for any momentum $p$ and $\delta_{\mu \mu}=D$.

\section{B Flow vertices}

The flow vertices $X^{(2,0)}, X^{(3,0)}$ and $X^{(1,1)}$ are defined through eqs. (2.13) and (6.12), respectively. They are explicitly given by

$$
\begin{aligned}
X^{(2,0)}(p, q, r)_{\mu \nu \rho}^{a b c}= & i f^{a b c}\left\{(r-q)_{\mu} \delta_{\nu \rho}+2 q_{\rho} \delta_{\mu \nu}-2 r_{\nu} \delta_{\mu \rho}\right. \\
& \left.+\left(\alpha_{0}-1\right)\left(q_{\nu} \delta_{\mu \rho}-r_{\rho} \delta_{\mu \nu}\right)\right\}, \\
X^{(3,0)}(p, q, r, s)_{\mu \nu \rho \sigma}^{a b c d}= & f^{a b e} f^{c d e}\left(\delta_{\mu \sigma} \delta_{\nu \rho}-\delta_{\mu \rho} \delta_{\sigma \nu}\right) \\
& +f^{a d e} f^{b c e}\left(\delta_{\mu \rho} \delta_{\sigma \nu}-\delta_{\mu \nu} \delta_{\rho \sigma}\right)+f^{a c e} f^{d b e}\left(\delta_{\mu \nu} \delta_{\rho \sigma}-\delta_{\mu \sigma} \delta_{\nu \rho}\right), \\
X^{(1,1)}(p, q, r)_{\mu}^{a b c}= & \alpha_{0} i f^{a b c} r_{\mu} .
\end{aligned}
$$

In the Feynman diagrams, the momenta in these formulae are identified with the ingoing line momenta. 


\section{Evaluation of the diagrams in figure 5}

The contributions of the diagrams in figure 5 to the Fourier transform of the correlation functions in the BRS identity (6.23) are of the form

$$
-\frac{g_{0}^{4} i f^{a b c}}{\lambda_{0} p^{2} q^{2} r^{2}} \mathcal{C}_{\mu, n}
$$

where $n$ is the diagram number. Explicitly, one obtains

$$
\begin{aligned}
& \mathcal{C}_{\mu, 1}=\frac{1}{2} p^{2}(q-r)_{\mu} \mathrm{e}^{-t \alpha_{0}\left(q^{2}+r^{2}\right)}+\frac{1}{2}\left(q^{2}-r^{2}\right) p_{\mu} \mathrm{e}^{-t \alpha_{0} p^{2}}-\mathcal{C}_{\mu, 2}, \\
& \mathcal{C}_{\mu, 2}=\frac{1}{2}\left\{p^{2}(q-r)_{\mu}+\left(q^{2}-r^{2}\right) p_{\mu}\right\} \mathrm{e}^{-t p^{2}},
\end{aligned}
$$

for the diagrams contributing to the left-hand side of the equation and

$$
\begin{aligned}
& \mathcal{C}_{\mu, 3}=-p^{2} r_{\mu} \mathrm{e}^{-t \alpha_{0}\left(q^{2}+r^{2}\right)}, \\
& \mathcal{C}_{\mu, 4}=-\frac{1}{2} p^{2} p_{\mu}\left\{\mathrm{e}^{-t \alpha_{0}\left(q^{2}+r^{2}\right)}-\mathrm{e}^{-t \alpha_{0} p^{2}}\right\}, \\
& \mathcal{C}_{\mu, 5}=-\left(q r+r^{2}\right) p_{\mu} \mathrm{e}^{-t \alpha_{0} p^{2}},
\end{aligned}
$$

for the diagrams on the right-hand side. The BRS symmetry requires

$$
\mathcal{C}_{\mu, 1}+\mathcal{C}_{\mu, 2}=\mathcal{C}_{\mu, 3}+\mathcal{C}_{\mu, 4}+\mathcal{C}_{\mu, 5}
$$

which is indeed the case.

Open Access. This article is distributed under the terms of the Creative Commons Attribution Noncommercial License which permits any noncommercial use, distribution, and reproduction in any medium, provided the original author(s) and source are credited.

\section{References}

[1] M. Lüscher, Properties and uses of the Wilson flow in lattice QCD, JHEP 08 (2010) 071 [arXiv: 1006.4518] [SPIRES].

[2] M. Lüscher, Topology, the Wilson flow and the HMC algorithm, PoS (Lattice 2010) 015 [arXiv: 1009.5877] [SPIRES].

[3] J. Zinn-Justin, Renormalization and stochastic quantization, Nucl. Phys. B 275 (1986) 135 [SPIRES].

[4] J. Zinn-Justin and D. Zwanziger, Ward identities for the stochastic quantization of gauge fields, Nucl. Phys. B 295 (1988) 297 [SPIRES].

[5] C. Becchi, A. Rouet and R. Stora, Renormalization of the abelian Higgs-Kibble model, Commun. Math. Phys. 42 (1975) 127 [SPIRES].

[6] C. Becchi, A. Rouet and R. Stora, Renormalization of gauge theories, Annals Phys. 98 (1976) 287 [SPIRES].

[7] K. Symanzik, Schrödinger representation and Casimir effect in renormalizable quantum field theory, Nucl. Phys. B 190 (1981) 1 [SPIRES]. 